\title{
preprint
}

\section{Bistability, Autowaves and Dissipative Structures in Semiconductor Fibers with Anomalous Resistivity Properties}

\author{
Eduard G. Karpov* \\ Department of Civil \& Materials Engineering, University of Illinois, Chicago, IL 60607
}

(Received 00 Month 200x; final version received 00 Month 200x)

\begin{abstract}
This work provides a discussion of bistability conditions, switching autowaves properties and emergence of dissipative structures in semiconducting fibers with anomalous positive dependence of electrical resistivity on temperature of a sigmoid type, $\left(1+e^{-T}\right)^{-1}$. An open system thermodynamics approach is utilized for the analysis of this dissipative solid-state system. The approach aims to represent the structure of solution space of its governing equation in the form of physical phase diagrams, known as nonequilibrium phase diagrams, and two specific binary diagrams have been obtained here. One of the diagrams, where electrical power density and ambient temperature represent external parameters, shows a wide region with dissipative structures as nonuniform steady state temperature profiles on the fiber. A possibility of efficient external control over dissipative structure geometry is also demonstrated.
\end{abstract}

Keywords: open system, autowave, dissipative structure, nonequilibrium phase diagram, barium titanate

\section{Introduction}

A significant volume of recent research publications point out on the ability of inorganic systems to demonstrate some generic forms of emerging synergetic behavior that were earlier attributed to living organisms only. This behavior is represented by the processes of temporal evolution, self-organization and complication in highly inequilibrium physical systems given a sufficient inflow of energy and building material. According to the open systems theory $[1,2]$, these processes are accompanied by entropy exchange between the system and ambient media. Highly inequilibrium conditions in the system created by inward and outward energy flux may trigger autonomous formation of some stable nonuniform patterns on the spatial or temporal profiles of system parameters. In a closed system, such patterns could only be attributed to transient, rather than stationary processes. Examples of such stable patterns in open systems can be very broad, from current oscillations in electric generator circuits and oscillatory chemical reactions, to Benard cell vortices in convective flow of heated liquids. With the formation of stable nonuniform patterns, known as dissipative structures $[2,3]$, the open system undergoes a transition to a new physical phase often allowing for a greater energy throughput compared to the states with uniform system parameters. Such transitions are sometimes called nonequilibrium phase transitions and the system demonstrating those, the active system or active medium. In the context of dissipative structures, the phenomenon

*Email: ekarpov@uic.edu 


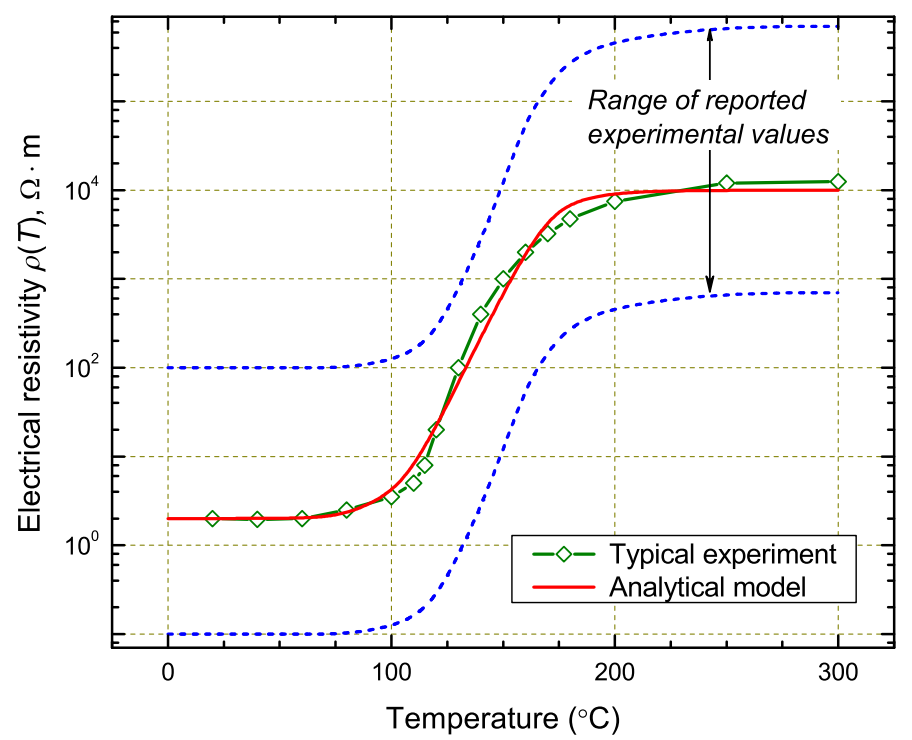

Figure 1. Temperature dependence of barium titanate ceramics with PTCR property. Anomalous sharp increase starts in the vicinity of $120^{\circ} \mathrm{C}$ Curie point. Solid line shows the averaged analytical model studied here.

of autonomous organization and complication is not a unique feature of the biological world; it may also be attributed, possibly in simpler forms, to various inorganic systems.

One basic type of active systems is the bistable or trigger systems [2-9]. Constitutive elements of a bistable system are found in one of two possible states or phases which are stable to small changes of external conditions. External effect of a magnitude grater than some finite threshold value may trigger a nonequilibrium phase transformation in one or several constitutive elements; that in turn may lead to a synergistic transition of the entire bistable system from one global stable phase to another. In this paper we explore conditions on bistability, nonequilibrium phase transitions, and emergence of dissipative structures in a simple type of material systems: resistively heated prismatic semiconductor fibers with a highly nonlinear positive dependence of electrical resistivity on temperature. Our discussion utilizes an open system thermodynamics approach. Here, the final goal is to represent solution space structure of nonlinear governing equations for this open dissipative system in the form of physical phase diagrams, using both analytical and numerical techniques.

\section{Anomalous Resistivity of Some Semiconducting Ceramics}

Barium titanate based ceramics can be given as one example of semiconductor materials that demonstrate a very interesting property of anomalous increase of electrical resistivity for temperatures near the Curie point being $120^{\circ} \mathrm{C}$ for pure $\mathrm{BaTiO}_{3}$, see Figure 1. This effect is uncommon for semiconductor materials and is called the positive temperature coefficient of resistivity (PTCR). The pure barium titanate is electrically insulating, but can be transformed into a semiconductor with the Verwey's controlled valency method [10]. The method is based on a partial (0.1$0.3 \%$ ) replacement of barium $\mathrm{Ba}_{2}^{+}$ions with trivalent donor or pentavalent acceptor ions. For example, addition of $\mathrm{La}_{3}^{+}$donors leads to $\mathrm{Ba}_{1-x} \mathrm{La}_{x} \mathrm{TiO}_{3}$ semiconducting ceramics. 
The PTCR effect is very pronounced for yttrium and strontium modified barium titanate ceramics [11-16], for samples doped with niobium [10, 17, 18], holmium [19], strontium, lanthanum [14], niobium pentoxide, yttrium hexaboride [15, 16], some oxygen-free compounds [20], and for other types of barium titanate ceramics [21] obtained with various synthesis procedures [15, 16, 21, 22]. According to reference [10] this phenomenon can be explained by the formation of barrier layers at the grain boundaries at higher temperatures, and was more recently demonstrated by direct observations of temperature dependence of these potential barriers [18]. Positive temperature coefficient thermistors called posistors utilizing the barium titanate based semiconductors are used in practice as temperature sensors, heating elements and current protection devices.

\section{Analytical Model of Anomalous Resistivity}

Resistivity of a typical barium titanate ceramics with PTCR property has a temperature dependence of the type shown in Figure 1. At temperatures below $100^{\circ} \mathrm{C}$, the resistivity $\rho$ is about constant or has a very weak dependence on temperature, while on the interval $100-200^{\circ} \mathrm{C}$, the $\rho$ value increases dramatically by three to five orders of the magnitude, followed by another region of weak temperature dependence after $200^{\circ} \mathrm{C}$. An example experimental curve $\rho(T)$ adopted from [15] is shown in Figure 1, while an entire family of reported experimental dependencies $[10,13-16,18,19,22-24]$ is quite diverse, and its approximate boundaries are shown on the same plot with dash lines.

For the purpose of analytical and numerical studies below in this paper, we approximate an experimental curve from [15] with a smooth analytical function of the logistic sigmoid type,

$$
\rho(T)=\frac{1}{\left(\rho_{\max }-\rho_{\min }\right)^{-1}+e^{-a\left(T-T_{0}\right)}}+\rho_{\min }
$$

Here, $a=0.12 \mathrm{~K}^{-1}$ is a steepness parameter; value $T_{0}=95^{\circ} \mathrm{C}$ determines the transition onset; $\rho_{\min }=2 \Omega \cdot \mathrm{m}$ and $\rho_{\max }=10^{4} \Omega \cdot \mathrm{m}$ are asymptotic values of the function at low and high temperatures, respectively. This function has a single inflexion point at $171.8^{\circ} \mathrm{C}$ for the selected values of parameters. In Figure 1, analytical model (1) is plotted in comparison to the experimental data.

\section{Energy Balance and Governing Equations}

We consider a long prismatic fiber made of a barium titanate ceramics type material whose electrical resistivity dependence on temperature is described by the equation (1). Assume a circular cross section of area $A$ and perimeter $p=2 \sqrt{\pi A}$, see Figure 2 (inset). Two ends of the fiber are connected to an electric power source to maintain a uniform current density $j=I / A$ in the longitudinal direction $x$ of the fiber. Consider a differential element of the fiber of length $d x$. During an infinitesimal time interval $d t$, resistive Joule heating of this element yields volume distributed thermal energy of a magnitude $j^{2} A \rho(T) d x d t$. This received energy is partially stored in the element volume, $\mu c_{p} A d T d x$, where $\mu$ and $c_{p}$ are mass density and specific heat capacity of the fiber material, and partially released convectively through the side surface of the element. The latter can be expressed using Newton's formula for the convective heat transfer, $\left(T-T_{\infty}\right) p h d x d t$, where $T_{\infty}$ is the ambient temperature. Finally, assuming the Fourier law for conductive heat flux in longi- 


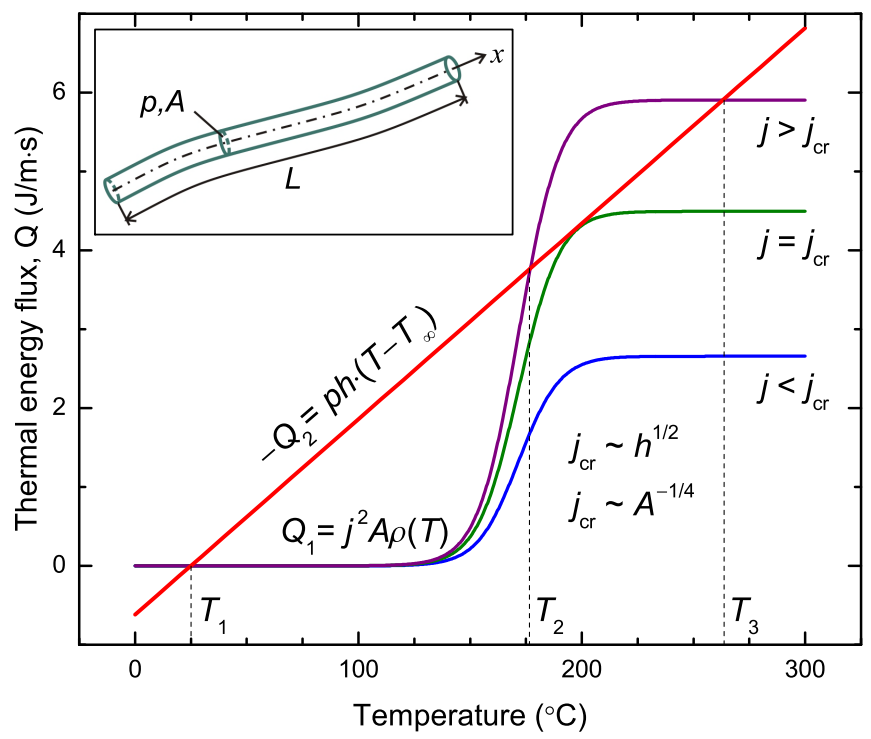

Figure 2. Energy exchange terms $Q_{1}$ and $Q_{2}$ in governing equation (3) as functions of temperature at various current densities.

tudinal direction of the fiber, $-k A \nabla T$, where $k$ is a heat conduction coefficient, elemental energy balance equation can be written as

$$
j^{2} A \rho(T) d x d t-\left.k A \frac{\partial T}{\partial x}\right|_{x} d t=\mu c_{p} A d T d x+\left(T-T_{\infty}\right) p h d x d t-\left.k A \frac{\partial T}{\partial x}\right|_{x+d x} d t
$$

This equation can be rearranged in terms of energies per unit time and unit fiber length to give a heat transfer PDE for the temperature function $T=T(x, t)$ with an essentially nonlinear righthand side,

$$
\begin{aligned}
& \mu c_{p} A \dot{T}-k A \frac{\partial^{2} T}{\partial x^{2}}=f(T), \quad f(T)=Q_{1}(T)+Q_{2}(T) \\
& Q_{1}(T)=j^{2} A \rho(T), \quad Q_{2}(T)=p h \cdot\left(T_{\infty}-T\right)
\end{aligned}
$$

where $\rho$ is the resistivity function (1). Reported values of mass density for barium titanate are in the range $5.83-6.02 \mathrm{~g} / \mathrm{cm}^{3}[25,26]$, thermal conductivity 2.59-2.89 W/(m.K) [26] and specific heat 400-450 $\mathrm{J} /(\mathrm{kg} \cdot \mathrm{K})[26,27]$ at room temperature. These parameters are quite uniform in the temperature range 20$300^{\circ} \mathrm{C}$, though the specific heat shows an increase of about $10-20 \%$ near the Curie point $[26,27]$. For the analysis below, we average this data and select $\mu=5.92 \mathrm{~g} / \mathrm{cm}^{3}, k=2.74 \mathrm{~W} /(\mathrm{m} \cdot \mathrm{K}), c_{p}=480 \mathrm{~J} /(\mathrm{kg} \cdot \mathrm{K})$. Temperature dependence of specific heat is ignored as insignificant compared to that of electrical resistivity. Convection heat transfer coefficient $h$ and ambient temperature $T_{\infty}$ are set at $7.0 \mathrm{~W} / \mathrm{m}^{2} \cdot \mathrm{K}$ and $25^{\circ} \mathrm{C}$, respectively. Wire cross section area $A=1.0 \mathrm{~mm}^{2}$, and the current density $j$ will be varied. In some cases we will also consider fibers with 0.5 and $0.25 \mathrm{~mm}^{2}$ cross sections for comparison.

\section{Qualitative Discussion: Monostability and Bistability}

In this section we discuss interesting qualitative properties of solutions to the governing equation (3). This equation is written in units of thermal power per unit 
length of the fiber. Therefore, the nonlinear function $Q_{1}(T)$ is the axial density of power generated in the fiber due to Joule heating, and the linear function $Q_{2}(T)$ represents power dissipated by the fiber to the ambient due to convection. Assume the convection coefficient $h$, fiber cross section area $A$ and perimeter $p$ are set constant as the internal (system) parameters, while the ambient temperature $T_{\infty}$ and electric current density $j$ are viewed as the controlled external parameters. Depending on a specific realization of these two parameters, the transcendental equation

$$
f(T)=Q_{1}(T)+Q_{2}(T)=0
$$

may normally have one $\left(T_{1}\right)$ or three $\left(T_{1-3}\right)$ roots, see Figure 2 , being the fiber temperatures at which the generated Joule heat is balanced by convective heat dissipation. Since heat exchange between the fiber and external bodies is absent at each of these temperatures, an initial condition of the type $T(x, 0)=T_{i}(i=1$, 2 or 3 ) for the governing PDE (3) will yield a stationary solution $T(x, t)=T_{i}$ for any $x$ and $t$, which will be stable for $T_{1}$ or $T_{3}$ and unstable for $T_{2}$. The slope of function $Q_{1}(T)$ is smaller than that of $-Q_{2}(T)$ at points $T_{1}$ and $T_{3}$, but greater at the point $T_{2}$, see Figure 2. Thus, for a small increase of temperature about value $T_{1}$ or $T_{3}$, heat dissipation $\left(-Q_{2}\right)$ prevails over heat generation $\left(Q_{1}\right)$, while for the value $T_{2}$ heat generation prevails on the contrary. In other words, any small fluctuation of temperature in a fiber segment about $T_{1}$ or $T_{3}$ tends to be mitigated, while any small deviation about point $T_{2}$ tends to be magnified. Noteworthily, equilibrium temperatures $T_{1-3}$ are not altered for a constant ratio $A / p h$, or $A / h^{2}$ for circular cross sections, which represents compensation of a surface effect due to linear increase of the fiber cross section area with a quadratic increase of heat transfer through side surface of the fiber.

Monostability. The usual situation of resistive fiber heating in convective media is observed for currents small enough, such that $Q_{1}$ and $-Q_{2}$ intersect at one point only, see Figure 2. This point represents the only possible thermal equilibrium of the system with the ambient, as in the case of usual fibers with a constant or monotonously varying resistivity. This standard situation is referred to as monostability. According to (4), the system is monostable at currents smaller than some critical value $j_{c r}$, which depends on the heat transfer coefficient and fiber cross section area as $j_{c r} \sim h^{1 / 2}$ and $j_{c r} \sim A^{-1 / 4}$, respectively. In particular, $j_{c r}=21.15 \mathrm{~A} / \mathrm{m}^{2}$ for the selected value $T_{\infty}=25^{\circ} \mathrm{C}$. Furthermore, there exists a minimal current, $j_{\min }=9.11 \mathrm{~A} / \mathrm{m}^{2}$, such as the slope of $Q_{1}$ at the saddle point is equal to the slope $-Q_{2}$. If the supply current is smaller than $j_{\text {min }}$, the system is monostable for any ambient temperature. Since the ambient temperature $T_{\infty}$ gives a vertical shift to the $-Q_{2}$ line, the latter will intersect with $Q_{1}$ at one point only if $T<T_{\infty}^{\min }$ or $T>T_{\infty}^{\max }$, where $T_{\infty}^{\min }$ and $T_{\infty}^{\max }$ are some critical ambient temperatures for each given $j>j_{\text {min }}$. For example, values $T_{\infty}^{\min }=25.0^{\circ} \mathrm{C}$ and $T_{\infty}^{\max }=138.2^{\circ} \mathrm{C}$ correspond to the current magnitude $21.15 \mathrm{~A} / \mathrm{m}^{2}$ mentioned earlier. In summary, the system is monostable if the ambient temperature is beyond the interval $\left[T_{\infty}^{\min }, T_{\infty}^{\max }\right]$ or the current density drops below $9.11 \mathrm{~A} / \mathrm{m}^{2}$.

Bistability. Triple intersection of functions $Q_{1}$ and $-Q_{2}$ at sufficiently low ambient temperatures and currents $j>j_{c r}$, Figure 2, represents a qualitatively different physical behavior of the system. Increase of the current density beyond $j_{c r}$ leads to emergence of two additional states of thermal equilibrium at $T(x, t)=T_{2}$ and $T(x, t)=T_{3}$. The system is now bistable having two stable equilibrium states, $T_{1}$ and $T_{3}$, and one absolutely unstable equilibrium state, $T_{2}$. Any small perturbation of state $T_{2}$ will trigger a fast transition of the entire system into one of the sta- 


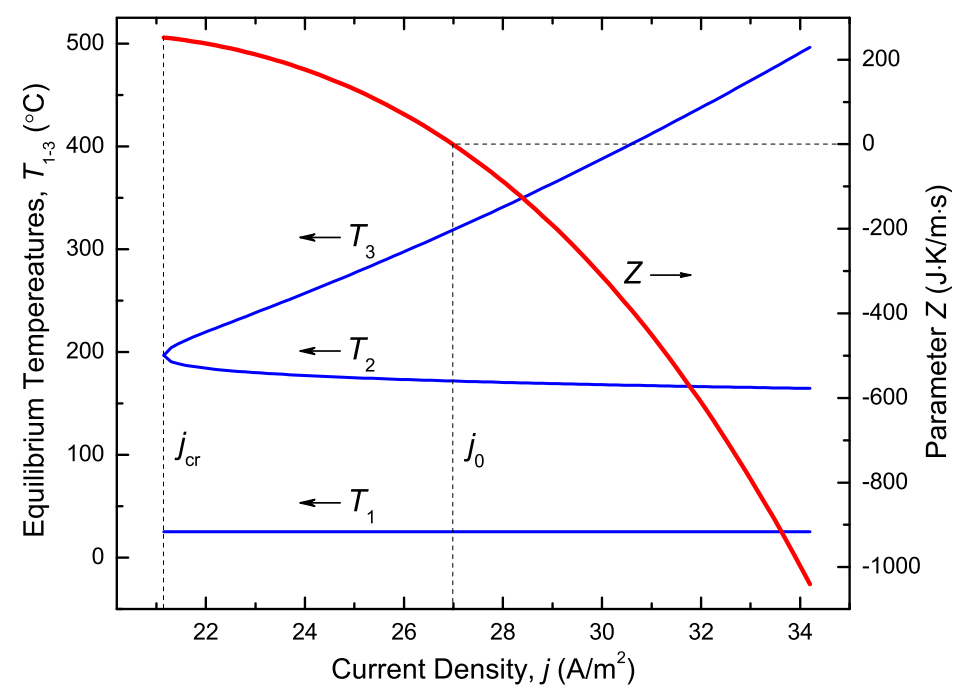

Figure 3. Dependencies of $Z$-integral and equilibrium temperatures $T_{1-3}$ on current density $j$ at ambient temperature $T_{\infty}=25^{\circ} \mathrm{C}$. Special values: $j_{c r}=21.15 \mathrm{~A} / \mathrm{m}^{2}, j_{0}=26.98 \mathrm{~A} / \mathrm{m}^{2}$

ble states, $T(x, t)=T_{1}$ or $T(x, t)=T_{3}$. On the contrary, transition between the states $T_{1}$ and $T_{3}$ would require a large perturbation of a magnitude greater than the difference $T_{2}-T_{1}$ or $T_{2}-T_{3}$ and a significant longitudinal span. This type of transformations between two stable configurations of an open system is known as nonequilibrium phase transition [1,2]. Insufficiently large perturbations will relax to the original stable state, see Figure 4. Furthermore, any initial profile of the type $T(x, 0)<T_{2}$ for all $x$ may only relax to $T(x, t)=T_{1}$, and a profile $T(x, 0)>T_{2}$ for all $x$ will always relax to $T(x, t)=T_{3}$ at $t \rightarrow \infty$.

For currents just above $j_{c r}$, values $T_{2}$ and $T_{3}$ are close to each other, and the state $T_{3}$ can be viewed as metastable. In other words, transitions from hot to cold state is easier than the reverse in this case. Further increase of current density $j$ beyond the value $j_{0}=26.98 \mathrm{~A} / \mathrm{m}^{2}$ corresponding to the condition $Z\left(j_{0}, T_{\infty}\right)=0$, where

$$
Z\left(j, T_{\infty}\right)=-\int_{T_{1}}^{T_{3}} f(T) d T
$$

will transform $T_{3}$ into an absolutely stable state, while $T_{1}$ will become a metastable state. Numerical studies of Section 6 prove that value $j_{0}$ serves as a threshold current density, after which a cold-to-hot transition of the system is easier to initiate than the reverse one. Therefore, we may consider the $Z$-integral (5) as a function of external parameters $j$ and $T_{\infty}$, which is indicative of whether the hot state is metastable in the system $(Z>0)$, or the cold state is metastable instead $(Z<0)$. Dependencies of value $Z$ and equilibrium temperatures $T_{1-3}$ on current density $j$ at $T_{\infty}=25^{\circ} \mathrm{C}$ are drawn in Figure 3 plot. We may also see from (1) and (5) that, for a constant ratio $A / h^{2}$, the magnitude of $Z$ is directly proportional to $A$.

Critical nucleus. Consider the situation, when hot state $T_{3}$ is absolutely stable, and cold state $T_{1}$ is metastable. Assume an initial condition that the fiber is found mostly in the $T_{1}$ state, except for a small region $\delta L$ at temperature $T_{3}$, a hot phase "nucleus". Small perturbations of this type will relax back to the metastable state $T_{1}$, while those with $\delta L$ greater than some critical value $\delta L_{c r}$ will trigger 


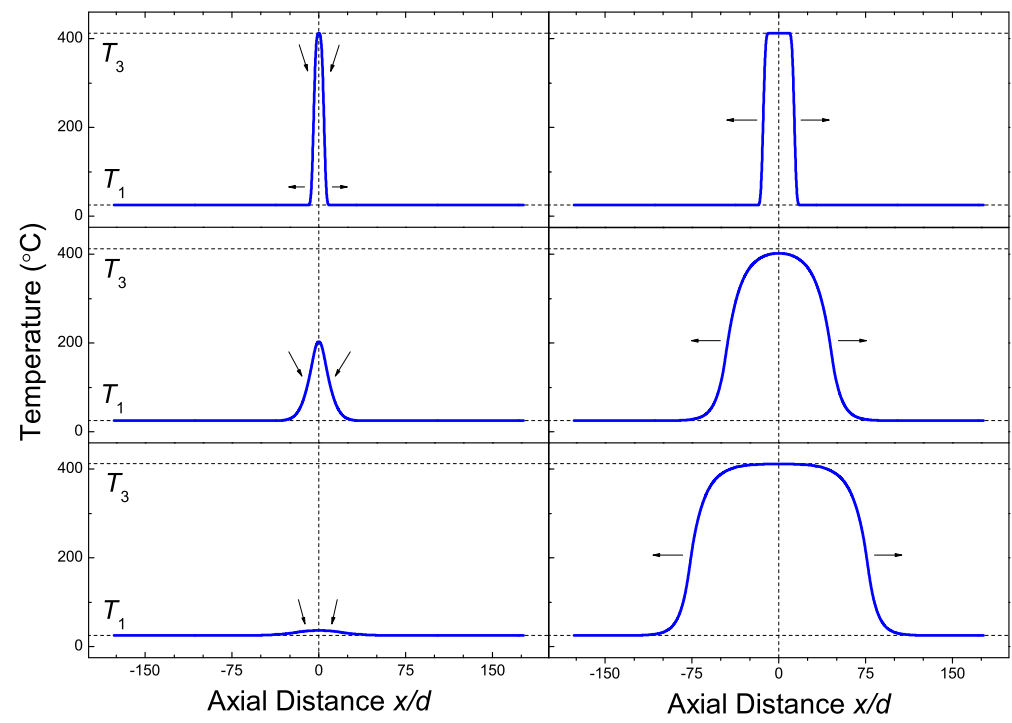

Figure 4. Evolution of small (left) and large (right) perturbations of the equilibrium state $T_{1}$; parameter $d$ is the fiber diameter.

transition of the entire system to the state $T_{3}$, see Figure 4 and Figure 5 inset. If the external parameters $\left(j, T_{\infty}\right)$ are maintained constant, left and right boundaries of the initial hot phase nucleus will somewhat disperse due to conductive heat exchange in the fiber and then start moving relative to their original positions at a finite velocity, as illustrated in Figure 4.

Autowaves. The process of widening of a hot phase nucleus can be viewed as two progressive autowaves moving in the opposite directions, whose passage is switching the system from cold to hot state. These autowaves represent a special transient solution to (3) of the type $T=T(x \pm c t)$, where $c$ is the autowave front velocity. A solution with $c=0$ would correspond to a very interesting situation of long-term co-existence of both cold and hot regions in the fiber. The system will demonstrate similar behavior for the reverse situation, where $T_{1}$ is the absolutely stable state. In both cases, determination of the critical nucleus size and autowave front velocity are interesting tasks that cannot be performed analytically even for the present, relatively simple nonlinearity of function $f(T)$ in equation (3). Obviously, $\delta L_{c r}$ and $c$ should depend on $Z$ and therefore on the external parameters $T_{\infty}$ and $j$, while specific quantitative dependencies may only be determined numerically, as discussed below in Section 6. Quantitative information about $\delta L_{c r}$ and $c$ will also be useful in summarizing system properties in the form of nonequilibrium phase diagrams. Another type of system behavior, different to that described in Figure 3, can be expected when only a limited amount of electric power is supplied to the system. Indeed, widening of the $T_{3}$ zone results in a dramatic increase of Joule heat dissipation in the fiber that may by mitigated with a limited or fixed power supply. This situation is also investigated numerically in Section 7.

\section{Numerical Studies}

Euler method. A standard finite element method procedure [28, 29], can be utilized to reduce the nonlinear heat transfer equation (3) into a first order ODE in time 
written in a matrix form,

$$
\mu c_{p} A L \dot{\mathbf{T}}+\mathbf{K} \mathbf{T}=Q(\mathbf{T}), \quad Q(\mathbf{T})=j^{2} A L \rho(\mathbf{T})+p h L\left(\mathbf{T}_{\infty}-\mathbf{T}\right)
$$

where $L$ is the length of each of the axial finite elements, or distance between the FEM nodes, $\mathbf{T}$ is a vector of nodal temperatures, so that $T_{n}$, the $n$-th element of $\mathbf{T}$, is the temperature at node $n$, and $\mathbf{K}$ is the FEM conductance matrix. For a periodic boundary condition $T_{0}=T_{N_{e}}$, where $N_{e}$ is the total number of elements, K is a circular $N_{e} \times N_{e}$ matrix of the type

$$
\mathbf{K}=\frac{k A}{L}\left(\begin{array}{ccccc}
2 & -1 & 0 & \ldots & -1 \\
-1 & 2 & -1 & \ldots & 0 \\
0 & -1 & 2 & \ldots & 0 \\
\ldots & \ldots & \ldots & \ldots & \ldots \\
-1 & 0 & 0 & \ldots & 2
\end{array}\right)
$$

Time integration of the nonlinear matrix equation (6) can be performed numerically using Euler method,

$$
\mathbf{T}^{(i+1)}=\mathbf{T}^{(i)}+\frac{\Delta t}{\mu c A L}\left(Q\left(\mathbf{T}^{(i)}\right)-\mathbf{K} \mathbf{T}^{(i)}\right), \quad i=1,2, \ldots, N_{\Delta t}
$$

where $\Delta t$ is a numeral time step and $N_{\Delta t}$ is a total number of time steps.

Consider a prismatic fiber of a circular cross-section area with all the physical parameters listed in Section 4. Three various cross-section areas will be considered, $A=1.0 \mathrm{~mm}^{2}, 0.5 \mathrm{~mm}^{2}$ and $0.25 \mathrm{~mm}^{2}$. When studying $0.5 \mathrm{~mm}^{2}$ and $0.25 \mathrm{~mm}^{2}$ fibers in comparison with the $1.0 \mathrm{~mm}^{2}$ case, a decrease of the side surface area is compensated with a proportional decrease of the convection coefficient, $h \sim A^{1 / 2}$, to elucidate a pure geometric effect of fiber cross section area. Since transient processes occur faster in thinner fibers, the time step $\Delta t$ was set at $0.5 \mathrm{~s}$ for $1.0 \mathrm{~mm}^{2}$ cross section, while at $0.35 \mathrm{~s}$ and $0.25 \mathrm{~s}$ for the $0.5 \mathrm{~mm}^{2}$ and $0.25 \mathrm{~mm}^{2}$ cases, respectively. 20000 time steps and 160 finite elements of length $L=3 d$, where $d=2 \sqrt{A / \pi}$ is the fiber diameter, were utilized in all simulations.

$\left(j, T_{\infty}\right)$-parametrization. Consider current density $j$ provided by an external power supply and ambient temperature $T_{\infty}$ as controlled external parameters that are kept constant during an entire simulation run. The system behavior is analyzed with reference to various selected values $j$ and $T_{\infty}$.

Critical nucleus size $\delta L_{c r}$ was first investigated. Initial shape of the nucleus is shown in Figure 5 (inset), where $\delta s$ is the transient zone width set at $3 d$ in all cases. Current density $j$ varied from $j_{c r}=21.15 \mathrm{~A} / \mathrm{m}^{2}$ to $40-50 \mathrm{~A} / \mathrm{m}^{2}$, depending on $A$. Value $\delta L_{c r}$ was determined by setting first a $\delta L$ and then varying $j$ with $0.01 \mathrm{~A} / \mathrm{m}^{2}$ resolution to determine a minimal current that is still able to initiate the $T_{1} \rightarrow T_{3}$ transition in the entire fiber for the selected $\delta L$. The latter was then regarded as $\delta L_{c r}$ corresponding to the current value $j$. For the $T_{3} \rightarrow T_{1}$ transition the procedure was similar, except that the threshold current was defined as the maximal one at which the transition to the cold state is still occurring for the selected $\delta L$. Results of the calculations for three various cross section areas of the fiber and ambient temperature $25^{\circ} \mathrm{C}$ are presented in Figure 5. Remarkably, all curves on this plot have a common vertical asymptote at $j_{0}=26.98 \mathrm{~A} / \mathrm{m}^{2}$. Furthermore, $\delta L_{c r}$ appeared independent of a total fiber length.

Autowave traveling velocity was investigated by tracking motion of the saddle point on the autowave front, see Figure 4, on a similar series of simulations. The autowave velocity $c$ was constant during an entire simulation run, except for short 


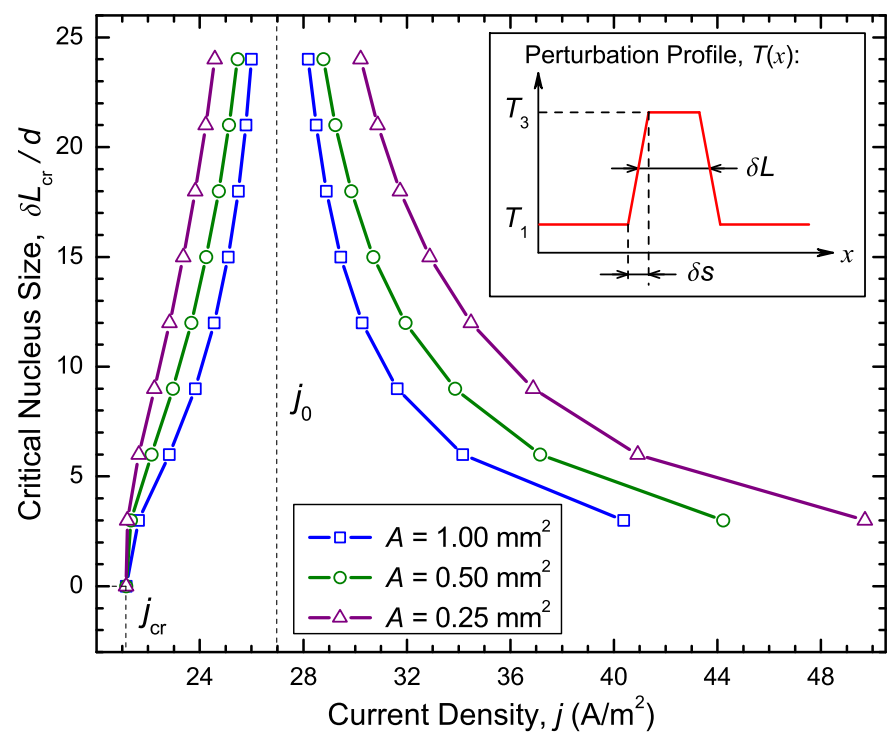

Figure 5. Minimal nucleus size required to trigger transition between the stable states at $T_{\infty}=25^{\circ} \mathrm{C}$ for various current densities and fiber cross-section areas. Inset shows nucleus profile for the $T_{1} \rightarrow T_{3}$ transition corresponding to the three curves on the right to $j=j_{0}$. Three curves on the left to $j_{0}$ correspond to the $T_{3} \rightarrow T_{1}$ transition, where the shown nucleus profile is reversed upside down.

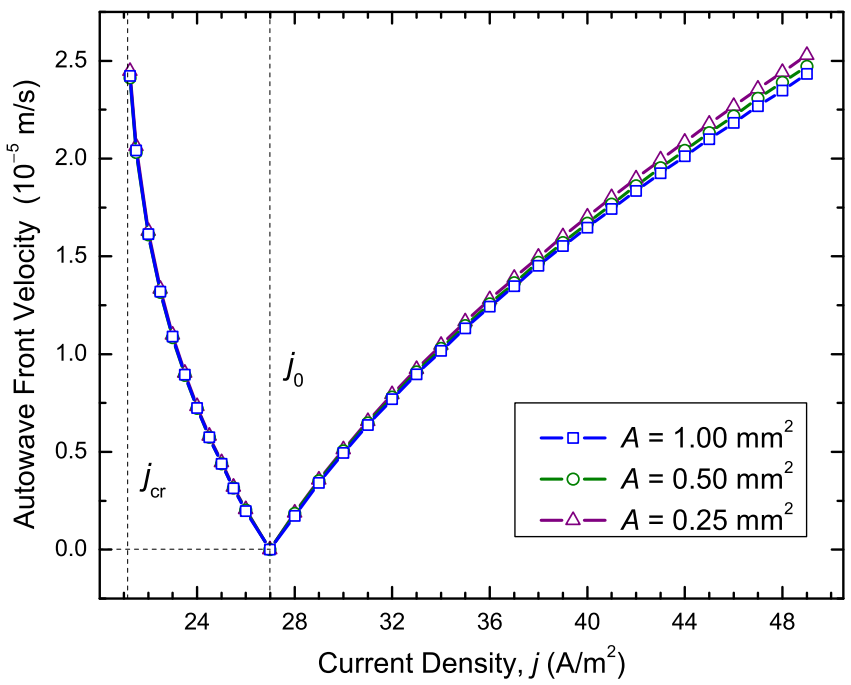

Figure 6. Autowave front velocity. At $j>j_{0}$ the autowave is moving as shown in Figure 4 (right), while at $j<j_{0}$ it is moving in the opposite direction corresponding to a reverse phase transition.

initial and final periods of time, when the autowave front is yet forming or already vanishing due to periodic boundary conditions. Interestingly, value $c$ was independent of total fiber length and initial nucleus size $\delta L$. Dependence on fiber cross section area was also weak, while current density was a major parameter affecting $c$, see Figure 6 . For the $T_{1} \rightarrow T_{3}$ transition $\left(j>j_{0}\right)$, this dependence is close to linear at high current densities. For the reverse transition $T_{3} \rightarrow T_{1}\left(j<j_{0}\right)$, $c$ is very large for small currents in the vicinity of $j_{c r}$ and then decays exponentially when the current grows toward the $j_{0}$ value. The most important result of 


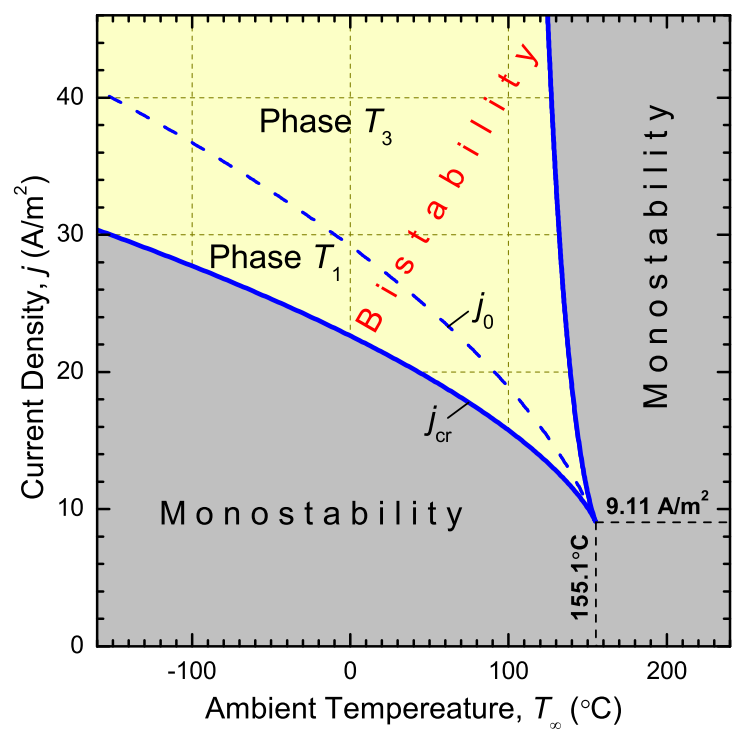

Figure 7. $\left(j, T_{\infty}\right)$-type phase diagram of the system. A definite boundary between the phases $T_{1}$ and $T_{3}$ exists (dash line), when initial condition contains seeds of the both phases. Upon relaxation of transient processes, the entire bistable system is either in $T_{1}$ or $T_{3}$ state.

the autowave studies is the fact that front velocity $c$ is never zero, unless current density is close to the value $j_{0}$. When current magnitude is passing this value, the autowave propagation is changing its direction to the opposite.

$\left(j, T_{\infty}\right)$-type phase diagram. We may conclude that for the $\left(j, T_{\infty}\right)$ parametrization, the cold and hot phases generally do not co-exist in the system. The only exception is the current value $j_{0}$ associated with $Z=0$ and leading to a zero autowave velocity. If the initial condition $T(x, 0)$ contains at least one critical nucleus of each phase and $j \neq j_{0}$, only a limited time is required for the entire system to take one of the stationary configurations $T(x, t)=T_{1}$ or $T(x, t)=T_{3}$. A non-zero temperature gradient in the fiber may only be observed in the vicinity of physical boundaries at $t \rightarrow \infty$, and the entire system will take one of the uniform states: $T_{1}$ at $j<j_{0}$, or $T_{3}$ at $j>j_{0}$. Thus, we are able to draw a phase diagram of the system with $j$ and $T_{\infty}$ serving as external control parameters, see Figure 7. As discussed earlier in Section 5, the overall bistability region on this diagram is defined by the conditions $T_{3}=T_{2}$ and $T_{1}=T_{2}$ for the bottom and top $T_{\infty}$ limits, respectively. Numerical solution of the respectful transcendental equations involving $f(T)$ from $(3)$, where $T_{\infty}$ is viewed as unknown parameter for a selected $j$, gives two converging curves depicted in Figure 7 with solid lines. Boundary between the two stable phases in the regime of bistability (dash line) is defined by the condition (5). All three curves terminate on the single branching point $\left(9.11 \mathrm{~A} / \mathrm{m}^{2}, 155.1^{\circ} \mathrm{C}\right)$, which is somewhat analogous to a triple point on a usual equilibrium phase diagram. Since boundaries between different regimes and phases are fully determined by the behavior of function $f(T)$ in equation (3), the entire $\left(j, T_{\infty}\right)$-diagram is invariant to heat conduction coefficient $k$, mass density $\mu$ and specific heat capacity $c_{p}$ of the fiber material. These parameters may only affect characteristics of transient processes in the systems, such as critical nucleus size, autowave velocity and relaxation times. The present $\left(j, T_{\infty}\right)$ phase diagram is therefore a fundamental characteristic of semiconducting PTCR fibers defined by the resistivity function (1). 


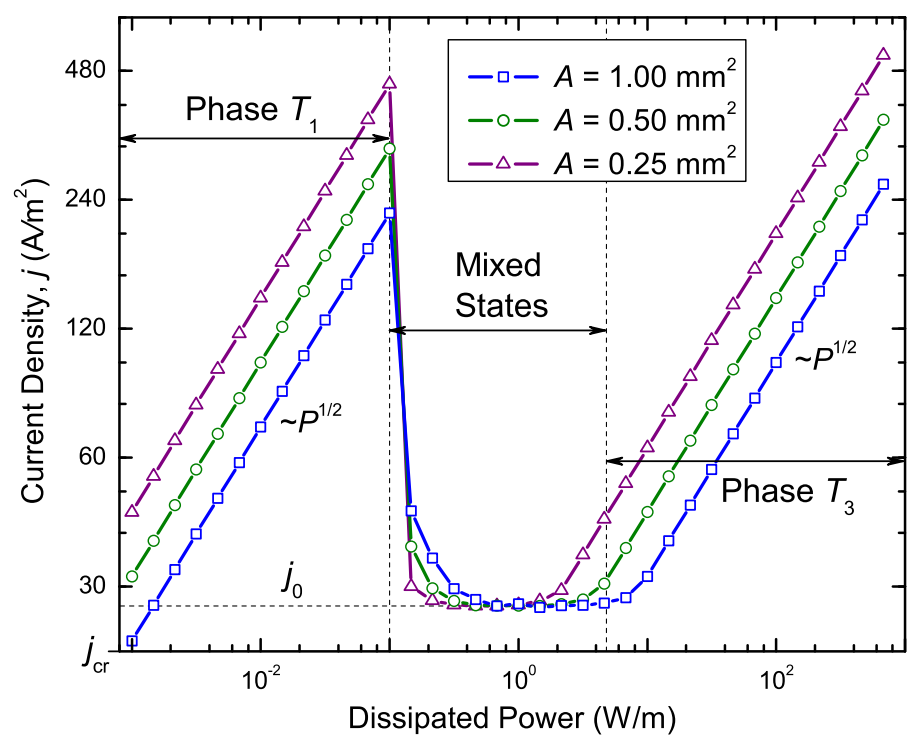

Figure 8. Dependence of steady state current density on the Joule power dissipated by the system.

\section{Stable Dissipative Structures}

Particularly interesting is the situation of long-term coexistence of two phases in the same system. This implies an intriguing possibility to create non-uniform temperature patterns, while keeping uniform the system geometry and ambient conditions. As shown in the previous section, this can be achieved with $\left(j, T_{\infty}\right)$ control parameters under a special condition $j=j_{0}$ leading to a zero propagation velocity of the autowave front. However, this situation is not practical due to the necessity to fix one of the external parameters, so that control over the temperature profile is limited to the variation of ambient temperature only. Furthermore, such a profile would have a neutral stability only, a tendency to be eliminated with a small fluctuation of external parameters and a strong dependence on initial conditions.

$\left(P, T_{\infty}\right)$-parametrization. Another type of system behavior can be realized for an alternative selection of external parameters, where inequilibrium temperature patterns are stable to small changes of these parameters and also invariable for a wide class of initial conditions. As mentioned earlier, such patterns are known as dissipative structures $[2,3]$. Consider the situation when ambient temperature and electric power, rather than current density, delivered to the fiber are chosen as fixed external parameters. If they are maintained constant, one may expect that hot phase expansion by autowave motion and resultant increase of fiber resistance will be mitigated with the limited power supply. As current magnitude self-adjusts to the supplied power level, evolution of a hot phase zone will stabilize on the fiber. With numerical studies below in this section we elucidate details of this expected behavior and discuss a $\left(P, T_{\infty}\right)$-type phase diagram of the system in comparison with the $\left(j, T_{\infty}\right)$ diagram developed earlier. Possibilities of external control over geometric properties of dissipative structures are also demonstrated.

Current density. Numerical studies of the $\left(P, T_{\infty}\right)$-parameterized system began with investigation of dependence of steady state current density $j$ (at $t \rightarrow \infty$ ) on the value $P$ being the supplied electric power divided by total fiber length. The $j$-to- $P$ dependence appeared in a very interesting form presented in Figure 8 for $T_{\infty}=25^{\circ} \mathrm{C}$. For powers smaller than $0.10 \mathrm{~W} / \mathrm{m}$, the stationary state current grows 


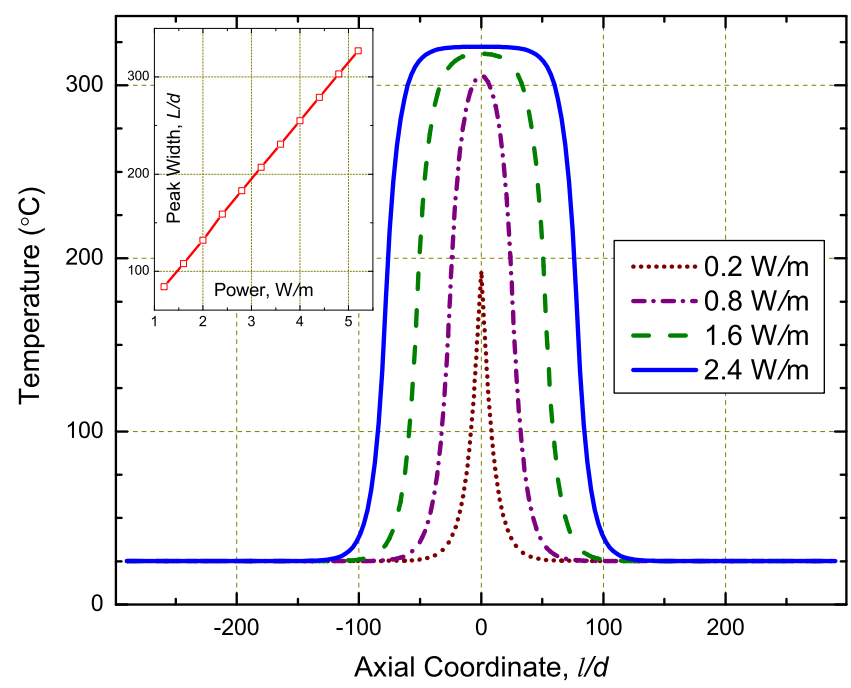

Figure 9. Dissipative structure shape at various $P$ and $T_{\infty}=25^{\circ} \mathrm{C}$.

as $P^{1 / 2}$, reaching a value of several hundred $\mathrm{A} / \mathrm{m}^{2}$ depending on fiber's crosssection area $A$. Despite of such large currents, much exceeding $j_{c r}$ and even $j_{0}$, stationary temperature profiles were uniform at $T_{1}$ for any $P<0.1 \mathrm{~W} / \mathrm{m}$, even if initial conditions contained seeds of the hot phase $T_{3}$. In other words, such powers are insufficient to maintain a nonuniform temperature pattern; the entire system remains in the cold phase, because occurrence of any hot phase island would lead to a dramatic drop of fiber total conductance and subsequent decrease of current density below the $j_{0}$ value. For a range of powers from about 0.15 to $9.0 \mathrm{~W} / \mathrm{m}$, the current density has a plateau where $j$ is close to $j_{0}$ for all such powers; a more specific position of this plateau depends on $A$. Beyond the $9.0 \mathrm{~W} / \mathrm{m}$ power level, the current density again grows in a standard $P^{1 / 2}$ manner.

Dissipative structures. Studies of temperature profiles for the range of $P$ from 0.15 to $9.0 \mathrm{~W} / \mathrm{m}$ corresponding to the anomalous behavior of current density shown in Figure 8 indicate that stationary non-uniform temperature patterns are generated in the system at these conditions. These patterns demonstrate all basic features of stable dissipative structures. In particular, they always have shapes of standing autowaves as shown in Figure 4, i.e. their presence is equivalent to co-existence of both cold and hot phases on the same fiber. Furthermore, any small region on the fiber with initial temperature greater than $T_{\infty}$ (seed of the hot phase) evolves into a dissipative structure, whose shape at $t \rightarrow \infty$ is determined by values of external parameters only. Typical shapes of stationary temperature profiles for this dissipative structure at various $P$ and $T_{\infty}=25^{\circ} \mathrm{C}$ are shown in Figure 9 . Variation of parameter $P$ only changes width of the structure, while its hight (temperature $T_{3}$ ) remains close to a constant value. Figure 9 inset plot shows that this dependence is very closely linear for the range of powers $1.2-5.2 \mathrm{~W} / \mathrm{m}$. In the absence of any boundary effects on a sufficiently long fiber, shape of dissipative structures is also invariant to the total fiber length.

When two identical seeds of the hot phase $T_{3}$ are provided by initial conditions and distance between them is smaller than width of the transition zone between phases $T_{1}$ and $T_{3}$ on a stationary temperature profile, these seeds will evolve into a single dissipative structure of the same type as shown in Figure 9. If the distance 


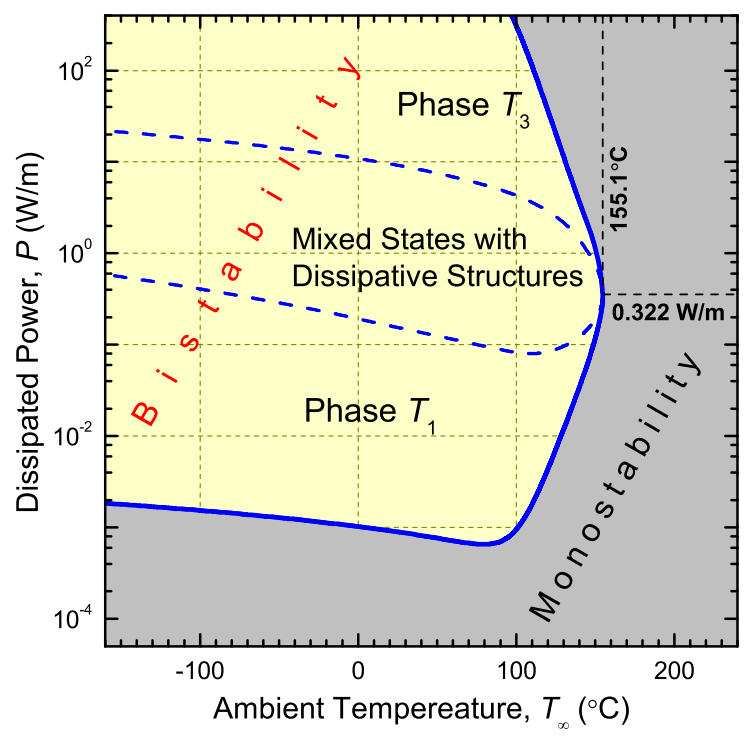

Figure 10. $\left(P, T_{\infty}\right)$-type phase diagram of the system.

between two seeds is very large compared to the transition zone width, they evolve into two narrower dissipative structures that remain stable indefinitely long. Linear growth of the hot zone width with the supplied power $P$, see Figure 9 inset, provides means of efficient control over dissipative structure evolution. Since total resistance of the fiber also grows linearly with the peak width, voltage control over dissipative structures can be alternatively utilized.

$\left(P, T_{\infty}\right)$-type phase diagram. As found earlier in this section, $\left(P, T_{\infty}\right)$-type parametrization of external conditions leads to formation of stable dissipative structures in the system with nonzero temperature gradients along the fiber length. Emergence of these structures is equivalent to existence of a standing autowave, and therefore long-term coexistence of both cold and hot phases in the system. At ambient temperature $T_{\infty}=25^{\circ} \mathrm{C}$, dissipative structures are formed if the supplied electric power is in the range $0.15-9.0 \mathrm{~W} / \mathrm{m}$. Further numerical studies of the system at various ambient temperatures showed emergence of dissipative structures for $T_{\infty}$ from at least $-160^{\circ} \mathrm{C}$ until the value $155.1^{\circ} \mathrm{C}$, see Figure 10 . Beyond this temperature the system becomes monostable, similar to the case of $\left(j, T_{\infty}\right)$ diagram in Figure 7. Power values corresponding to the region with dissipative structures are somewhat greater at lower temperatures, and their range is narrowing fast in the vicinity of the $155.1^{\circ} \mathrm{C}$ point. Overall boundary of the bistability region is still entirely determined by the $f(T)$ function behavior, as for the $\left(j, T_{\infty}\right)$ diagram, though in a somewhat indirect manner. Namely, values $P$ on this boundary at given $T_{\infty}$ were calculated as $j^{2} A \rho(T)$, where $j$ is equal to $j_{c r}$ from the $\left(j, T_{\infty}\right)$ diagram, and $T$ is one of the stable equilibrium temperatures $\left(T_{1}\right.$ or $\left.T_{3}\right)$ corresponding to the selected ambient temperature and current. Thus, the overall bistability region is invariant to the system parameters $k, \mu$ and $c_{p}$. Meanwhile, the region of mixed states shown with a dash line in Figure 10 will depend on the thermal conductivity coefficient $k$ in equation (3). For instance, if $k$ was smaller than value $2.74 \mathrm{~W} /(\mathrm{m} \cdot \mathrm{K})$ chosen here, the system would allow for narrower dissipative structures with shaper edges, and therefore the region of mixed states would span wider on the power axes at all ambient temperatures. 


\section{Conclusion}

We conclude that open systems involving PTCR semiconducting fibers with anomalous resistivity characteristic of a logistic type (1) depicted in Figure (1) demonstrate bistability for a wide range of external parameters, such as ambient temperature and supplied electric current or power. When the ambient temperature $T_{\infty}$ and current magnitude $j$ are fixed as external parameters, the entire system is found in one of the stable phases after all transient processes are relaxed. Special transient processes in the form of autowave propagation can be triggered by introducing to the system a nucleus of an opposite phase whose critical size has been investigated. Propagation of the autowaves, whose front velocity has been discussed as well, leads to switching of the system to an opposite stable state. A nonequilibrium phase diagram of the $\left(j, T_{\infty}\right)$-parameterized system has been developed.

Even more interesting behavior was observed when the ambient temperature $T_{\infty}$ and supplied electric power $P$ were selected as controlled external parameters. In this case, spatial dissipative structures in the form of stable patterns with nonzero temperature gradients emerge in the system for a well-defined range of external parameters. As a result, the $\left(P, T_{\infty}\right)$-type phase diagram features a large region where cold and hot phases co-exist on the same fiber indefinitely long. Shape and size of the dissipative structures are invariant to initial conditions and most system parameters, and the size can be controlled in a linear manner by changing the parameter $P$. For powers large enough to maintain dissipative structures, current density in the fiber is almost constant for all such powers. In view of these findings, progressive autowave motion in the $\left(j, T_{\infty}\right)$-controlled system may also be interpreted as a process of evolution of newly formed dissipative structures under varying electric power supplied to the fiber. Generally, autonomous emergence of complexity in the form of dissipative structures studied here is a nonequilibrium phase transition enabling the system to dissipate much greater powers at smaller currents.

There is also a basic analogy with biological systems where population growth cannot be initiated before some minimal amount of food is provided to the system per unit time. When this condition is met the population grows to a maximal size mostly defined by the food supply rate. Similarly, thermal dissipative structures discussed here emerge after some threshold electric power is provided and then stabilize their growth on a stationary configuration reflecting the given power supply condition.

One interesting task for the future could be demonstration of pulsating (spatiotemporal) dissipative structures in similar systems through implementation of a nonlinear resistivity function with retarded response properties.

\section{Acknowledgements}

Support of this research by National Science Foundation via grant \#0900498 is gratefully acknowledged.

\section{References}

[1] H. Haken Information and Self-Organization: A Macroscopic Approach to Complex Systems, Springer, Berlin, 2006.

[2] G. Nicolis and I. Prigogine Self-Organization in Nonequilibrium Systems, Wiley, New York, 1977.

[3] Y.L. Klimontovich Statistical Theory of Open Systems, Vol.1, Kluwer Academic Publishers, Dordrecht, Netherlands, 1995.

[4] X. Xin, Journal of Dynamics and Differential Equations 3 (1991) p.541-573. 
[5] P. Hanggi, H. Grabert, P. Talkner and H. Thomas, Physical Review A 29 (1984) p.371-378.

[6] S. Bouzat and H. Wio, Physics Letters A 268 (2000) p.323-329.

[7] M. Berdau, G.G. Yelenin, A. Karpowicz, M. Ehsasi, K. Christmann and J.H. Block, Journal of Chemical Physics 110 (1999) p.11551-11573.

[8] D. Bedeaux, P. Mazur and R.A. Pasmanter, Physica 86 (1977).

[9] P. Mazur and D. Bedeaux, Journal of Statistical Physics 24 (1981) p.215-233.

[10] W. Heywang, Journal of Materials Science 6 (1971) p.1214-1226.

[11] P.H. Xiang, H. Harinaka, H. Takeda, T. Nishida, K. Uchiyama and T. Shiosaki, Journal of Applied Physics 104 (2008) p.094108.

[12] S.R. Syrtsova, V.N. Shuta, I.F. Kashevicha, A.S. Sedlovskya and B.E. Watts, Materials Science in Semiconductor Processing 5 (2003) p.223-225.

[13] P. Bomlai and N. Sirikulrat, Journal of Materials Science 40 (2005) p.779784.

[14] P.L. Janega, Solid-State Electronics 29 (1986) p.5966.

[15] X.X. Wang, H.L.W. Chan, G.K.H. Pang and C.L. Choy, Materials Science and Engineering B 100 (2003) p.286-291.

[16] X. Wang, H. Chan and C. Choy, Journal of the European Ceramic Society 24 (2004) p.12271231.

[17] S.H. Yoon and H. Kim, Journal of Applied Physics 92 (2002) p.1039-1047.

[18] M.W. Mancinia and P.I.P. Filho, Journal of Applied Physics 100 (2006) p.104501.

[19] A.J. Dekker Solid State Physics, Macmillan \& Co., London, 1970 Chapter 8: Ferroelectrics.

[20] L.L. Kovalenko, O.I. Vyunov and A.G. Belous, Journal of the European Ceramic Society 19 (1999) p.965-968.

[21] V.N. Shut and S.V. Kostomarov, Inorganic Materials 45 (2009) p.1417-1422.

[22] K. Park, J.G. Ha, C.W. Kim and J.G. Kim, Journal of Materials Science: Materials in Electronics 19 (2008) p.357-362.

[23] G.H. Jonker, Solid-State Electronics 7 (1964) p.895-903.

[24] M. Kuwabara, Journal of Applied Physics 76 (1994) p.1326-1328.

[25] D.R. Lide (editor) Handbook of Chemistry and Physics, 85th CRC Press, Boca Raton, FL, 2004.

[26] Y. He, Thermochimica Acta 419 (2004) p.135141.

[27] P. Supancic, Journal of the European Ceramic Society 20 (2000) p.2009-2024.

[28] D.L. Logan A First Course in the Finite Element Method, 4th Thomson, Toronto, ON, 2007.

[29] N.H. Kim and B.V. Sankar Introduction to Finite Element Analysis and Design, Wiley, New York, 2009. 